\title{
The Improvement of NetSolve System
}

\author{
Haiying Cheng ${ }^{1}$, Wu Zhang ${ }^{1}$, and Wenchao Dai ${ }^{2}$ \\ ${ }^{1}$ School of Computer Engineering and Science, Shanghai University, \\ Shanghai 200072, PRC \\ sunnychy@163.com, zhang@staff.shu.edu.cn \\ ${ }^{2}$ Dept. of Computer Science and Technology, East China Normal University, \\ Shanghai 200062, PRC \\ wenchaodai@163.com
}

\begin{abstract}
NetSolve is a kind of grid middleware used for high performance computing. In this article, the architecture and operational principle of NetSolve are first analyzed, the limitations of the Netsolve system are pointed out, and then Web Service Server and Server Proxy are put forward as the improvement of NetSolve System to solve these limitations.
\end{abstract}

\section{Introduction}

Thanks to advance in hardware, networking infrastructure and algorithms, computing intensive problems in many areas can now be successfully attacked. Various mechanisms have been developed to perform computations across diverse platforms. But until recently, there are still some difficulties in using high performance computer and grid technology on a large scale.

NetSolve, underway at the University of Tennessee and at the Oak Ridge National Laboratory, is a project that makes use of distributed computational resources connected by computer networks to efficiently solve complex scientific problems. This article is meant to probe into the implementation of NetSolve and describe its two extensions based on the super Cluster ZQ2000 of Shanghai University.

\section{NetSolve}

NetSolve is a client-server system that enables users to solve complex scientific problems remotely. The system searches for computational resources on the network, chooses the best one available, and solves a problem using retry for fault tolerance, and returns the answers to the user. It has three components:

- Agent: The agent maintains a database of NetSolve servers along with their capabilities and dynamic usage statistics for use in scheduling decisions. It attempts to find the server that will service the request, balance the load amongst its servers, and keep track of failed servers. 
- Server: The NetSolve server is a daemon process that awaits client requests. It can run on single workstations, clusters of workstations, SMPs, or MPPs. One key component of the server is the ability to wrap software library routines into NetSolve services by using an Interface Definition Language (IDL) .

- Client: The NetSolve client uses application programming interfaces (APIs) to make a request to the NetSolve system, with the specific details required with the request.

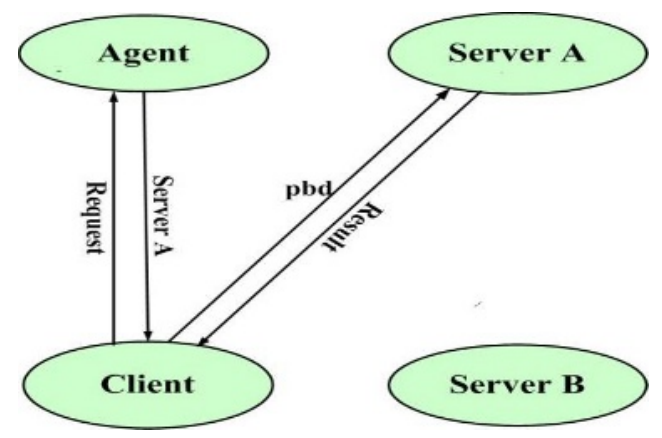

Fig. 1. NetSolve Architecture

\section{Limitations of the Netsolve System}

However, NetSolve system doesn't have a universal interface, its interface protocol can only be applied in the clients of NetSolve system. Therefore, other systems can't communicate with NetSolve directly. Besides, it can't provide a friendly and all-purpose interface for people who want to use the high performance computing resources. Another limitation is: unless clients, agent, servers are located in the same local network, real IP address is needed for them, as they communicate with each other via IP address. But for security and limitation of IP resources concern, many clusters, like ZQ2000 high performance cluster computer of Shanghai University, doesn't have real IP address for each node of the cluster. Therefore, NetSolve clients from outside the local network can't access any server on the node of ZQ2000.

To solve these limitations, we have two ways, the first is to make use of the Web Services technology to add a Web Services server into the NetSolve system, and the second is to add a server proxy into the NetSolve system.

\section{Web Services Server}

Web Services may use HTTP protocol, which can offer a friendly interface to facilitate calling services and make real IP address no longer a necessity. After adding a Web Services server into NetSolve, the work flow of the system changes as follows: 
(1) Developing tools that supporting Web Services can be used to query the service which exist in Web Services server. Service and its interface described in WSDL format are returned to users from the server. After that, clients can call the service, just like calling a common local process. (2) Clients send simple object access protocol requests to Web Services server. On receiving the requests, the Web Services server converts it into a service execution request to NetSolve system. NetSolve system can then select the best server to execute the corresponding service. (3) NetSolve system returns the results of a service to Web Services server after it is completed. Clients can then get simple object access protocol responses described in XML format from the Web Services server.

Web Services server turns services provided by NetSolve system into its services, which is needed by Web Services call interface. The Web Services server and NetSolve agent are installed on the same computer. All services provided by NetSolve servers are registered in NetSolve agent.

\section{Server Proxy}

The common structure of super-computer is that they have one or several pre-servers which possess real IP address and the rest fake IP address (local network IP address). The pre-servers have double IP address like Gateway. If we run a Server Proxy on pre-server or on Gateway, it can set up a bridge between the client and the interior NetSolve servers.

Server proxy must run on the pre-server or gateway of super-computer. It can ensure the success of connecting between client and server. First, client can connect to server proxy, because pre-server has real IP. Meanwhile, pre-server and interior server are in the same local area network (LAN), the pre-server can also connect to the interior servers. Another problem is that all the data must pass through pre-server, which may cause a bottleneck. Start up several server proxies in different pre-server, and each server proxy manage a few of the interior servers can solve this problem. Moreover, in order to improve the efficiency, we can set up a connection pool between the server proxy and the interior servers.

Agent manages server proxy. It has the right to kill server proxy. Server proxy registers to Agent at first time. If server proxy dies, Agent will delete the server proxy's information of the relevant interior servers.

\section{Conclusions}

These two kinds of improvement of NetSolve system have been successfully applied in the ZQ2000 high performance cluster computer of Shanghai University. There are many ways in which NetSolve should be further extended, we should constantly improve our system to catch up with the fast development of numerical computing. 


\section{References}

1. J. Dongarra, Network-enabled Solvers: A Step Toward Grid-based Computing, http:// www.siam.org/siamnews/12-01/solvers.htm

2. D. Arnold, Sudesh Agrawal, S. Blackford, and J. Dongarra. Users' Guide to NetSolve V1.4. Technical report, Computer Science Dept., University of Tennessee, May 2001. http://icl.cs.utk.edu/netsolve.

3. D.C. Arnold, D.Bachmann and J.Dongarra. Request Sequencing: Optimizing Communication for the Grid. [J].In Euro-Par 2000-Parallel Processing, August 2000.

4. H. Casanova and J. Dongarra, Applying NetSolve's network enabled server, IEEE Comput. Sci. \& Eng., 5:3 (1998), 57-66. 